\title{
Effectiveness of the biomechanical factors related to triple jump performance prediction during COVID 19 period in sri lanka
}

DOI: $10.46932 /$ sfjdv3n1-104

Received in: Jan 30st, 2021

Accepted in: Feb 1th, 2022

\author{
Pramesh Chathushka Thotawaththa \\ Temporary Demonstartor, (BSc. (Hons) in Sport Sciences and Management \\ Sabaragamuwa University of Sri lanka \\ Mr. P. C. Thotawaththa Temporary Demonstrator, \\ Department of Sports Sciences and Physical Education, \\ Faculty of Applied Sciences, \\ Sabaragamuwa University of Sri Lanka, \\ P.O.Box 02, Belihuloya, \\ Sri Lanka. \\ E-mail: pthotawaththa@gmail.com
}

\author{
A. W. S. Chandana \\ 1Department of Sport Sciences and Physical Education, Faculty of Applied Sciences, Sabaragamuwa \\ University of Sri Lanka, P. O. Box 02, Belihuloya, 70140, Sri Lanka. \\ E-mail: surajchandana@appsc.sab.ac.lk
}

\begin{abstract}
The triple jump is an athletic event consisting of three phases which are hop, step, and jump. According to the reversibility training method, that reveals when these athletes can't be able to maintain their physical fitness and performance properly, it influences for their performance negatively. This study was to identify how the athletes maintain the performances and how they obtain the performance prediction using the biomechanical method during the COVID-19 period in Sri Lanka. Those data were collected from five National standard male triple jumpers. The mean age of the participants is 26 . The triple jump technique was done by using the dynamic equation which included kinematic variables for flight phase of the above three phases. The Matlab17 software was used to optimize the flight phase. Three cameras $(100 \mathrm{~Hz})$ were used to observe the coordinates of center of mass and kinematics variables on the sagittal plane. The videos were analyzed through the Kinovea ( 0.9 .3 version) software. The hop dominated balance technique (35.5: 30.4: 34.1) was used for the prediction. The previous performances values of the players were $14.32 \mathrm{~m}$ to $16.07 \mathrm{~m}$ (Before 2020). Current COVID-19 period performances were $13.13 \mathrm{~m}$ to $15.43 \mathrm{~m}$. The velocity and angle were optimized by $5 \%$ and $+2^{0}$ outcome of the players were more than $16 \mathrm{~m}$. Considering this study, athletes' phases weren't in the optimum phase ratio. The hop dominated balance performance prediction and players' current and previous performances were significantly different in this study. Through this research all coaches and athletes can identify their shortcoming phase and values of the optimization variables and prediction performance level. If not, coaching techniques and tactics can be modified.
\end{abstract}

Keywords: Kinematics, Phase ratio, Prediction of performance, Triple Jump. 


\section{INTRODUCTION}

The triple jump is one of the tracks and field events, which requires the jumper to repeat the generation of maximal force to maintain the horizontal velocity during all phases of the jump. The triple jump consists of a running approach, 3 take-off phases in which the athlete hops on one foot, lands on the same foot, steps onto the opposite foot, and finally jumps and lands in the sand pit (Miladinov O, 2004). Triple jump distance is depended on the jumper's ability to apply the basic architectural paths during each of the 3 take-off phases (Yu, 1999). During each take-off phase a change in the movement structure and rhythm occurs, which affects the timing of each concentric and eccentric contraction (Koh \& Hay, 1990). Therefore, each take-off phase has its own dynamic requirements during the braking and propulsive phases.

In the triple jump, it is important that someone maintain the correct posture when jumping. Jumpers need to be conscious to keep their feet flat on the ground, as heel or toe jumping will negatively impact the jump. The triple jump is an athletics event where athletes combine speed, power, and agility to jump as far as possible from a starting point. There have been a number of attempts to determine the effect of phase ratio on triple jump performance using various approaches including observations of elite jumpers (Miller \& Hay,1999; Song \& Ryu, 2011); the differences between elite and novice jumpers (Simpson, 2007); statistical relationships between velocity tradeoffs during the contact phases (Yu \& Hay, 1999); and even an operations research approach (Brimberg, 2006).

Daily conditioning training may also help to reduce tension and stress from home confinement as a negative life event affects the mental health of elite athletes (Rice, Purcell , \& Silva, 2016). The abrupt change in their daily routine, the adopted home confinement measures, and uncertainty about the date for the return to activities can lead athletes to experience conditions that affect their mental health, such as external sources of distress, including financial problems, bad daily news, and internal sources of distress, such as worry about their performance when they return, and tension due to the routine change. This period can lead to negative feelings such as anxiety, depression, adverse behaviors, such as alcohol use and smoking, as well as eating and sleep disorders (Leonardo, Danilo, \& Alexandro , 2020). In this sense, to deal with these possible emotional concerns during the home confinement, coaches, strength and conditioning coaches, and athletes should pay attention to identifying and managing these experiences and seeking help and social support when necessary. Relaxation techniques, such as meditation, mindfulness, body scan, and deep breathing, are also recommended (Moore \& Gearity , 2019).

Attention to the dietary habits of athletes during home confinement is of comparable importance to exercise. This period may represent a sedentary moment for athletes, leading to consumption of high calorie foods due to impulse or anxiety (Rice, Purcell , \& Silva, 2016). In this sense, athletes should reduce 
caloric consumption. We recommend measures such as, if possible, reduction in macronutrient portion sizes; reduction, or even discontinuation, of the use of dietary supplements; prioritization of foods that can benefit the immune system (Rossi, Buford, McMillan, Kovacs, \& Marshall , 2010); and avoiding ultra-processed foods. In this sense, meals with an adequate protein intake, like meat or egg, sources of iron, zinc, and vitamin B12, with fruit and vegetables, sources of antioxidants, and vitamin C are recommended (Ormsbee \& Arciero, 2015).

In this COVID 19 situation, the training routines of a significant number of athletes around the world have been abruptly interrupted. Thus, sports science professionals and scientists are challenged to help athletes deal with some of these relevant aspects during this period. In the triple jump event, we train to improve lower limb muscle strength and other physical finesses. And the technique isn't followed correctly in due to this pandemic because lack of knowledge and lack of technical tools and poor knowledge about keeping the performance from dropping. This study was conducted to observe and investigate at the improvement and decline of triple jump athletes in the Covid-19 epidemic situation. By creating a model for every coach and athlete can understand the factors needed for their own fitness, achieve their highest potential and perform at their highest level. This study examines the coaches' biomechanical knowledge and the success of using it for training in such a situation.

\section{METHODOLOGY}

Triple jump players in Sri Lanka were taken as the population in this research study. This is due to the fact these Sri Lankan triple jump athletes have not demonstrated international level qualifications and cannot be properly trained during this COVID 19 pandemic period. There are three main stages in the triple jump event. Athletes can perform to the best of their ability by applying relevant balance techniques to those situations. This research was considered to find out the biomechanical predictions and the difference in performance of the athlete at the end of this period. It aims to optimize the distance of the players and correct them to improve the skills of the players and what happen the player performance. In this study, five of the best national triple jumpers in Sri Lanka were selected as the sample. The senior triple jumpers were chosen because it has a higher performance than the junior triple jumpers. Male triple jumpers show higher performance than female triple jumpers when comparing male and female triple jumpers. Therefore, this study focuses on male triple jumpers and this method is applicable to all triple jumpers in Sri Lanka. This study has selected the 5 best national senior male triple jumpers according to the 2019 National Championship in Sri Lanka and obtained data before the COVID period.

Two best performers were taken for the biomechanical optimization model. Player A (mass: 70 kg; height: $1.78 \mathrm{~m}$; best performance: $15.76 \mathrm{~m}$ ). Player $\mathrm{C}$ (mass: $71.35 \mathrm{~kg}$; height: $1.80 \mathrm{~m}$; best 
performance: $16.33 \mathrm{~m}$ ). Before the collection, the video data was done by pilot test in Sabaragamuwa university premises. After the forecast system, 3 more players are added, and their best performance is predicted and the performance drops during this period.

Optimization has done the takeoff velocity of the model was each increased by $3 \%, 4 \%$ and $5 \%$. In contrast, the takeoff angle $\left(-1^{0}, 0^{0},+1^{0},+2^{0}\right)$ from the measured values and all combinations of these two parameters were investigated, leading to 16 optimizations in total. The takeoff angle of the model was manipulated by increasing the three phases separately their phase distance. Approach velocities ranged from $8.1 \mathrm{~ms}^{-1}$ to $10.5 \mathrm{~ms}^{-1}$ (the maximum approach velocity recorded at the 2009 IAAF World Championships in Berlin [German Athletics Federation, 2009]). Optimization was used to maximize the distance of the whole triple jump in each condition.

$$
d_{\text {flight }}=v^{2} \sin 2 \theta / 2 g\left\{1+\left(1+2 g h / v^{2} \sin ^{2} \theta\right)^{1 / 2}\right\}(\text { Hay J. G., } 1992) \longrightarrow
$$

The above equation was used for distance during the flight phase. The optimize values were obtained as follows using the Matlab software for this equation (01). In the above equation, $\mathrm{h}$ was taken as a constant value and given as a mean value $(\mathrm{h}=0.1 \mathrm{~m})$ and the flight phase in the jump phase as $\mathrm{h}=$ $0.75 \mathrm{~m}$. Data related to the jump phase were taken from it. The data obtained in this way made it possible to obtain a large amount of data for velocity and angle (Thotawaththa \& Chandana, 2021).

\section{RESULTS}

\subsection{KINEMATICS VARIABLES}

The triple jump (without approach phase) of each athlete was captured by using 3 high-speed cameras $(100 \mathrm{~Hz})$, both on the sagittal planes. Through it, there were observed the coordination of all athletes. The collected data (mainly video clips, etc.) were analyzed by using the Kinovea software and there were found kinematic variables (Take off Velocity, etc.) (table 1,2,3). There has drawn and measured every frame COG using the Kinovea software. Then Find Below Variables in each player.

Table 1 Hop Phase Kinematics results

\begin{tabular}{cccc}
\hline & Hop Phase & & \\
\hline Parameters & Mean & SE Mean & SD \\
\hline TO Angle $(\theta)$ & 15.612 & \pm 0.49 & 1.55 \\
VV $\left(\mathrm{ms}^{-1}\right)$ & 2.2233 & \pm 0.0718 & 0.227 \\
HV $\left(\mathrm{ms}^{-1}\right)$ & 7.959 & \pm 0.105 & 0.331 \\
RV $\left(\mathrm{ms}^{-1}\right)$ & 8.266 & \pm 0.105 & 0.333 \\
TO Height $(\mathrm{m})$ & 1.188 & \pm 0.0178 & 0.0563 \\
Landing Height $(\mathrm{m})$ & 1.052 & \pm 0.0178 & 0.0563 \\
TO Distance $(\mathrm{m})$ & 0.4571 & \pm 0.0235 & 0.0742 \\
F Distance $(\mathrm{m})$ & 4.044 & \pm 0.122 & 0.385 \\
L Distance $(\mathrm{m})$ & 0.5947 & \pm 0.0101 & 0.0318 \\
Hop Distance $(\mathrm{m})$ & 5.096 & \pm 0.116 & 0.367 \\
\hline
\end{tabular}


Table 2 Step Phase Kinematics results

\begin{tabular}{cccc}
\hline & Step Phase & & \\
\hline Parameters & Mean & SE Mean & SD \\
\hline TO Angle $(\theta)$ & 11.875 & \pm 0.727 & 2.298 \\
VV $\left(\mathrm{ms}^{-1}\right)$ & 1.5085 & \pm 0.0911 & 0.2882 \\
HV $\left(\mathrm{ms}^{-1}\right)$ & 7.187 & \pm 0.12 & 0.38 \\
RV $\left(\mathrm{ms}^{-1}\right)$ & 7.349 & \pm 0.118 & 0.374 \\
TO Height $(\mathrm{m})$ & 1.141 & \pm 0.0172 & 0.0545 \\
Landing Height $(\mathrm{m})$ & 1.039 & \pm 0.00971 & 0.0307 \\
TO Distance $(\mathrm{m})$ & 0.5877 & \pm 0.0185 & 0.0586 \\
F Distance $(\mathrm{m})$ & 2.627 & \pm 0.0842 & 0.2661 \\
L Distance $(\mathrm{m})$ & 0.6634 & \pm 0.0224 & 0.071 \\
Step Distance $(\mathrm{m})$ & 3.878 & \pm 0.0625 & 0.1975 \\
\hline
\end{tabular}

Table 3 Jump Phase Kinematics results

\begin{tabular}{cccc}
\hline & Jump Phase & & \\
\hline Parameters & Mean & SE Mean & SD \\
\hline TO Angle $(\theta)$ & 20.6 & \pm 1.17 & 3.71 \\
VV $\left(\mathrm{ms}^{-1}\right)$ & 2.262 & \pm 0.122 & 0.386 \\
HV $\left(\mathrm{ms}^{-1}\right)$ & 6.026 & \pm 0.109 & 0.345 \\
RV $\left(\mathrm{ms}^{-1}\right)$ & 6.4488 & \pm 0.0963 & 0.3047 \\
TO Height $(\mathrm{m})$ & 1.248 & \pm 0.0142 & 0.0449 \\
Landing Height (m) & 0.528 & \pm 0.0231 & 0.073 \\
TO Distance (m) & 0.449 & \pm 0.0196 & 0.062 \\
F Distance (m) & 4.039 & \pm 0.114 & 0.36 \\
L Distance (m) & 0.444 & \pm 0.0382 & 0.1207 \\
Jump Distance (m) & 4.932 & \pm 0.122 & 0.387 \\
Full Distance (m) & 13.906 & \pm 0.228 & 0.72 \\
\hline
\end{tabular}

Looking at these results, the technology of these Sri Lankan players is at a very low level. There is also a $0.448306586 \%$ difference between the data obtained from the video analyzer and the actual distance. This study mainly provides an understanding of velocity and angle. In step and jump, the velocity drop is as low as $11 \%$ and $21.9 \%$. Athletes also use the maximum takeoff height and maximum takeoff angle relative to the hop and step for the maximum distance to avoid that velocity drop during the jump phase. The angle is maintained at $19^{0}-25^{\circ}$. It also maintains a very low take off angle during the step phase. It is a value between $8^{0}-15^{0}$. The triple jump event has three supportive phases. Spending more time on those three phases has a direct effect on the horizontal velocity. It can be seen by looking at the times of these players. The hop takeoff takes less time and more than two other supportive phases. According to the above data, it is obvious.

The Balance technique was used here. That is, its values are $35.5 \%, 30.4 \%$ and $34.1 \%$. Player A $14.15 \mathrm{~m}$ performance was divided according to the above ratio and values were obtained for hop, step, jump. It is shown in the table below. This was done by using the Matlab graphs as mentioned above to increase the velocity to $3 \%, 4 \%, 5 \%$ and keep it at an angle $\left(-1{ }^{0}, 0^{0},+1^{0},+2^{0}\right)$. Then the total distance was obtained as follows (table 4). 
Table 4 Optimized Simulation overall distance player A and C

\begin{tabular}{|c|c|c|c|c|c|}
\hline \multicolumn{6}{|c|}{ Optimized Simulation (Hop-Dominated Balance Technique) - Player A } \\
\hline & & \multicolumn{4}{|c|}{ Hop TO Angle $\left({ }^{0}\right)$} \\
\hline & & 14 & 15 & 16 & 17 \\
\hline & $100 \%$ & 13.5493 & 14.14085 & 14.70423 & 15.29577 \\
\hline Hop TO Velocity & $103 \%$ & 14.11268 & 14.76056 & 15.35211 & 15.97183 \\
\hline \multirow[t]{2}{*}{ Simulation } & $104 \%$ & 14.30986 & 14.92958 & 15.57746 & 16.19718 \\
\hline & $105 \%$ & 14.50704 & 15.15493 & 15.80282 & 16.4507 \\
\hline \multicolumn{6}{|c|}{ Optimized Simulation (Hop-Dominated Balance Technique) - Player C } \\
\hline & & \multicolumn{4}{|c|}{ Hop TO Angle $\left({ }^{0}\right)$} \\
\hline & & 14 & 15 & 16 & 17 \\
\hline & $100 \%$ & 14.76056 & 15.40845 & 16.02817 & 16.64789 \\
\hline Hop TO Velocity & $103 \%$ & 15.40845 & 16.08451 & 16.73239 & 17.40845 \\
\hline Simulation & $104 \%$ & 15.6338 & 16.30986 & 16.98592 & 17.66197 \\
\hline & $105 \%$ & 15.85915 & 16.56338 & 17.23944 & 17.94366 \\
\hline
\end{tabular}

Following results have shown the before COVID 19 Pandemic and during the pandemic results. And also, shown the above optimization values and performance drop presentation (table 5).

Table 5 Performance Variations

\begin{tabular}{ccccc}
\hline Player & $\begin{array}{c}\text { Previous } \\
\text { Performance(m) }\end{array}$ & $\begin{array}{c}\text { Current } \\
\text { Performance(m) }\end{array}$ & $\begin{array}{c}\text { Prediction } \\
\text { Performance(m) }\end{array}$ & $\begin{array}{c}\text { Performance Drop } \\
\text { \% }\end{array}$ \\
\hline A & 16.07 & 14.15 & 16.45 & 11.9 \\
B & 15.25 & 13.58 & 16.39 & 10.9 \\
C & 16.33 & 15.43 & 16.98 & 5.5 \\
D & 14.32 & 13.17 & 16.30 & 8.03 \\
E & 14.46 & 13.94 & 15.97 & 3.5 \\
\hline
\end{tabular}

\section{DISCUSSION}

To validate the designed biomechanical model all analyzed data were applied to the model of selected sample. Then the results of horizontal, vertical velocity and take off angle were calculated. Through it, optimization distance was calculated. That value of selected and phase ratio player A $37.3 \%$, $26.2 \%, 36.5 \%$. and player C $36.3 \%, 27.3 \%, 36.4 \%$. According to Jonathan Edward, the Phase ratio $35.5 \%, 30.4 \%, 34.1 \%$. for balance technique. The model shows the results increase velocity $3 \%, 4 \%, 5$ $\%$ and simulation above hop dominated current performance and balance technique used hop dominated optimization increase the overall performance.

Figure 1: (a) Techniques employed in Player A; Current simulation 14.15m, (b) Techniques employed in; Player C Current simulation $15.43 \mathrm{~m}$, (c) Techniques employed in; $17 \mathrm{~m}$ mark optimization simulation increased takeoff angles and takeoff velocities (Thotawaththa \& Chandana, 2021)

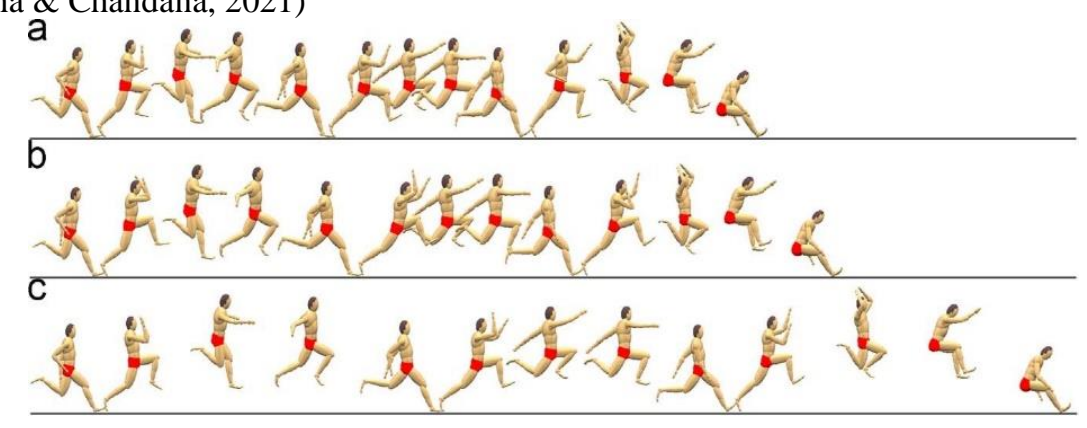


But Balance technique is the best results showed. Player A current performance $14.15 \mathrm{~m}$ after optimized $16.45 \mathrm{~m}$. Player C Current performance $15.43 \mathrm{~m}$ after optimized $16.98 \mathrm{~m}$. Current performance is very poor special step phase more force comes, after the hop phase that force, and velocity cannot balance next phase that's why step phase very poor. And CM rotation was not forward. Current player places the CM backward while supportive phase landing part. However, this model can be further developed to a 3D optimization model and performance variables optimization and kinematics and kinetic variable both add to this model can get a better result in applied biomechanics and sport practice which helps to optimize performance of triple jumpers. The values for the phases were obtained as follow from the above (figure 1) optimization. This shows that distance can be improved by balance technique optimization. Initial current optimization and hop dominated balance optimization method were taken to the consideration. Generally, two athletes' step phase was considerably less than other two phases.

Figure 2: Performance Variations

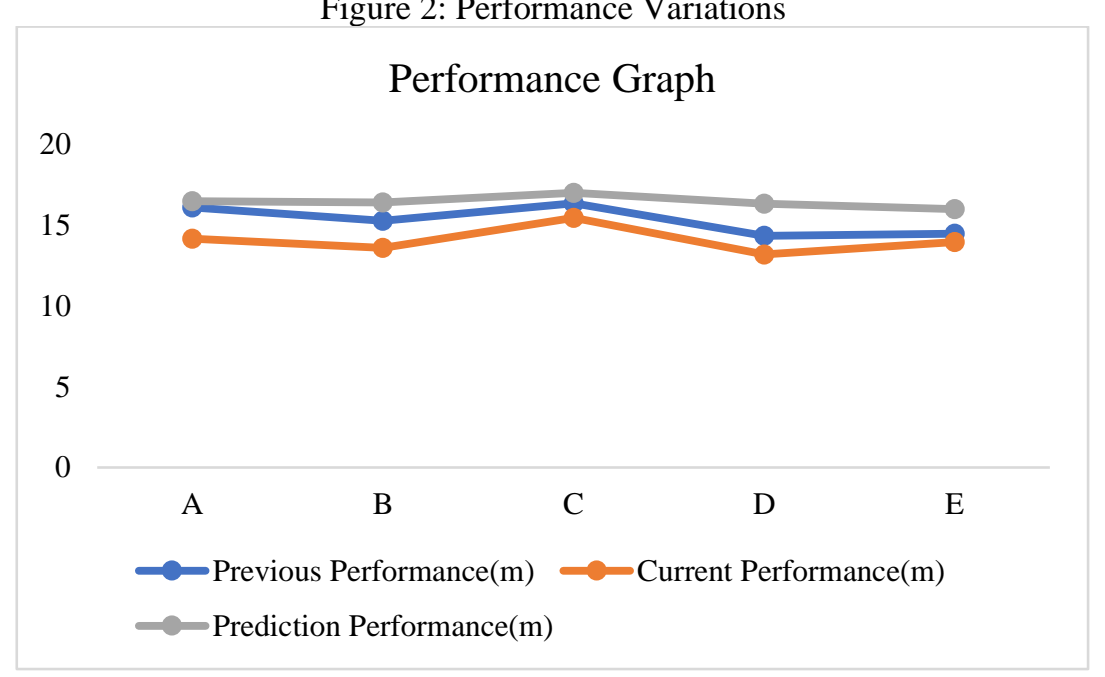

This study has found average of the drop percentage as $8 \%$. The previous performances are better than the current performances. These data were collected in after the isolation of Sri Lanka. Above graph (figure 2) has showed the prediction level and the current level. A conditioning routine can help athletes to boost and maintain immunity, minimize the effects of detraining, and facilitate the return to a normal routine, as well as improving tactical knowledge. Strength and conditioning professionals, teams, and athletes who able to adopt these measures will experience less difficulty when returning to their normal training and competition routines (Leonardo, Danilo, \& Alexandro, 2020).

\section{CONCLUSIONS}

Successful completion of this study, the model's all equations can be input to the software and after entering relevant data of any athlete, optimize velocity, takeoff angles and distance can be gain for 
each athletes / jumpers each phase. Through this research all coaches and athletes can identify their shortcoming phase and values of the optimization variables and prediction performance level. Apart from that, this biomechanical model can be used to improve the talent and to minimize the errors of postures when performing triple jump event. And also, this can be used to identify the relationship between the performance variables and the performance of the players. Further, the methodology that uses to create this model can be also used to create this type of models to other sports and other jumping events such as other vertical and horizontal jumping events. The performances of the players in Sri Lanka can be able to increase using the biomechanical training methods. Identifying the main variables, we can develop special training for that. In this case, following this prediction and find the performance level of the player can get ready for the upcoming competitions. And Sri Lankan Triple Jump Players can achieve more than $17 \mathrm{~m}$ mark and obtain Olympic medals. Coaches and Players should consider this biomechanical factors and models to develop the performances of the players. 


\section{REFERENCES}

1. Allen, S. (2009). Optimization and performance in the triple jump using computer simulation.

2. Bing, Y. (1996). Horizontal to vertical velocity conversion in the triple jump. Research Gate, 8995.

3. Chen , P., Mao, L., \& Nassis, G. (2020). Coronavirus disease (COVID-19): The need to maintain regular physical activity while taking precautions. Sport Health Sciences, 9, 103-104.

4. Hay, J. (1992). The biomechanics of the triple jump: a review. J. Sports Sci., 343-378.

5. Hay, J. (1999). Effort distribution and performance of Olympic triple jumpers. Journal of Applied Biomechanics, 36-51 .

6. Koh, T., \& Hay, J. (1990). Landing leg motion and performance in the horizontal jumps II. Journal of Sport Biomechanics, 361-373.

7. Leonardo, A. V., Danilo, C. R., \& Alexandro, A. (2020). Challeges to Athletics During the Home Confinement caused by the COVID-19 Pandemic. Strength and Conditioning Journal, 42, 1-5.

8. Miladinov O, B. P. (2004). Individual approach in improving the technique of triple jump for women. New Studies in Athletics, 27-36..

9. Moore, E., \& Gearity , B. (2019). Guest editorial for psychology of strength and conditioning special issue. Strength and conditioning, 41, 1-2.

10. Ormsbee, M., \& Arciero, P. (2015). Detraining increases body fat and weigth and decreases VO2 peak and metabolic rate. Strength and conditioning, 37, 72-83.

11. Rice, S., Purcell , R., \& Silva, S. (2016). The mental health of elite athletics: A narrative systematic review. Sports medicine, 46, 1333-1353.

12. Robert, G., Aron, J., Timothy, J., \& Xanne, J. A. (2011). Factors that differentiate Accelaration Ability in Field Sport Athletes. Journal of Strength and Conditioning Research, 25, 2704-2714

13. Simpson, R., Kunz, H., Agha, N., \& Graff, R. (2015). Exercise and the regulation of immune functions. Prog Mol Biol Transl Sciences, 135, 355-380.

14. Thotawaththa, P., \& Chandana, A. (2021). A Triple Jump Performance Optimization Model Based on Flight Phase Biomechanical Factors. IOSR Journal of Sports and Physical Education (IOSR-JSPE), 8(4), 10-17.

15. Yu, B. (1999). Biomechanical studies on triple jump techniques: theoretical considerations and applications. Proceedings of the 17th International Symposium on Biomechanics in Sports, 17-26

16. Yu, B. (1999). Horizontal-to-vertical velocity conversion in the triple jump. J Sports Science, 2219. 